PROCEEDINGS OF THE

AMERICAN MATHEMATICAL SOCIETY

Volume 132, Number 5, Pages 1377-1383

S 0002-9939(03)07244-7

Article electronically published on December 12, 2003

\title{
ON A SPECTRAL PROPERTY OF JACOBI MATRICES
}

\author{
S. KUPIN
}

(Communicated by Andreas Seeger)

\begin{abstract}
Let $J$ be a Jacobi matrix with elements $b_{k}$ on the main diagonal and elements $a_{k}$ on the auxiliary ones. We suppose that $J$ is a compact perturbation of the free Jacobi matrix. In this case the essential spectrum of $J$ coincides with $[-2,2]$, and its discrete spectrum is a union of two sequences $\left\{x_{j}^{ \pm}\right\}, x_{j}^{+}>2, x_{j}^{-}<-2$, tending to \pm 2 . We denote sequences $\left\{a_{k+1}-a_{k}\right\}$ and $\left\{a_{k+1}+a_{k-1}-2 a_{k}\right\}$ by $\partial a$ and $\partial^{2} a$, respectively.
\end{abstract}

The main result of the note is the following theorem.

Theorem. Let $J$ be a Jacobi matrix described above and $\sigma$ be its spectral measure. Then $a-1, b \in l^{4}, \partial^{2} a, \partial^{2} b \in l^{2}$ if and only if

$$
\text { i) } \int_{-2}^{2} \log \sigma^{\prime}(x)\left(4-x^{2}\right)^{5 / 2} d x>-\infty, \quad \text { ii) } \sum_{j}\left(x_{j}^{ \pm} \mp 2\right)^{7 / 2}<\infty .
$$

\section{INTRODUCTION}

It has been observed recently [4] that the sum rules [1, 2] for a Jacobi matrix $J$ lead to direct relations between elements of $J$ and its spectral properties. However, the sum rules become considerably more complicated when their order increases. In this note, we carry out a complete analysis of a sum rule of order six.

Let us consider a Jacobi matrix

$$
J=J(a, b)=\left[\begin{array}{ccc}
b_{0} & a_{0} & 0 \\
a_{0} & b_{1} & \ddots \\
0 & \ddots & \ddots
\end{array}\right],
$$

defined by two sequences $a=\left\{a_{k}\right\}, a_{k}>0$, and $b=\left\{b_{k}\right\}, b_{k} \in \mathbb{R}$. We suppose that $J$ is a compact perturbation of the so-called free Jacobi matrix $J_{0}$,

$$
J_{0}=\left[\begin{array}{ccc}
0 & 1 & 0 \\
1 & 0 & \ddots \\
0 & \ddots & \ddots
\end{array}\right]
$$

The (scalar) spectral measure $\sigma$ of $J$ is defined by the relation

$$
\left((J-z)^{-1} e_{0}, e_{0}\right)=\int_{\mathbb{R}} \frac{d \sigma(x)}{x-z},
$$

Received by the editors October 25, 2002.

2000 Mathematics Subject Classification. Primary 47B36; Secondary 42C05.

Key words and phrases. Jacobi matrices, sum rules. 
with $z \in \mathbb{C} \backslash \mathbb{R}$. It is well known that in this case the continuous part $\sigma_{c}$ of the measure is supported by $[-2,2]$, and its discrete part $\sigma_{d}$ lives on the union of two sequences $\left\{x_{j}^{ \pm}\right\}$such that $x_{j}^{+}>2, x_{j}^{-}<-2$, and $x_{j}^{ \pm} \rightarrow \pm 2$, as $j \rightarrow \infty$.

Let us put $\partial a=\left\{a_{k+1}-a_{k}\right\}$ and $\partial^{2} a=\left\{a_{k+1}+a_{k-1}-2 a_{k}\right\}$, and denote by 1 the sequence consisting of ones. The following theorem holds.

Theorem 0.1. Let $J=J(a, b)$ and $\sigma$ be its spectral measure. Then

$$
a-1, b \in l^{4}, \partial^{2} a, \partial^{2} b \in l^{2}
$$

if and only if

$$
\text { i) } \int_{-2}^{2} \log \sigma^{\prime}(x)\left(4-x^{2}\right)^{5 / 2} d x>-\infty, \quad \text { ii) } \sum_{j}\left(x_{j}^{ \pm} \mp 2\right)^{7 / 2}<\infty .
$$

The theorem has an immediate corollary.

Corollary 0.2. Let $J=J(a, b)$, and let $\sigma$ be its spectral measure. If $a-1, b \in$ $l^{4}, \partial a, \partial b \in l^{2}$, then relations 0.2 hold.

In this direction we also have (see [7]) that

$$
\text { i) } \int_{-2}^{2} \log \sigma^{\prime}(x)\left(4-x^{2}\right)^{3 / 2} d x>-\infty, \quad \text { ii) } \sum_{j}\left(x_{j}^{ \pm} \mp 2\right)^{5 / 2}<\infty,
$$

when $a-1, b \in l^{3}, \partial a, \partial b \in l^{2}$. Together with Corollary 0.2, this observation leads to a natural conjecture. The conjecture would yield a right discrete counterpart of results, obtained in [6] for Schrödinger operators on the half-line.

Conjecture 0.3. For an integer $k \geq 1$, conditions $a-1, b \in l^{k+1}, \partial a, \partial b \in l^{2}$ imply

$$
\text { i) } \int_{-2}^{2} \log \sigma^{\prime}(x)\left(4-x^{2}\right)^{k-1 / 2} d x>-\infty, \quad \text { ii) } \sum_{j}\left(x_{j}^{ \pm} \mp 2\right)^{k+1 / 2}<\infty .
$$

The author would like to thank B. Simon and P. Yuditskii for helpful discussions on the subject.

\section{Proof of Theorem 0.1}

It is convenient to map the domain $\mathbb{C} \backslash[-2,2]$ onto the unit disk with the help of the transformation $\zeta(z)=\frac{1}{2}\left(z-\sqrt{z^{2}-4}\right)$. We construct a measure $\mu$ on the unit circle by letting $d \mu(\theta)=d \sigma(x) /(2 \sin \theta)$, where $x=z\left(e^{i \theta}\right)$ and $z(\zeta)=\zeta+1 / \zeta$. The sequences $\left\{x_{j}^{ \pm}\right\}$are mapped to points $\left\{\zeta_{j}\right\}$, lying on the real diameter of the unit disk. In these new terms Theorem 0.1 says that $a-1, b \in l^{4}, \partial^{2} a, \partial^{2} b \in l^{2}$ if and only if

$$
\text { i) } \int_{0}^{2 \pi} \log \mu^{\prime}(\theta) \sin ^{6} \theta d \theta>-\infty, \quad \text { ii) } \sum_{j}\left(1-\left|\zeta_{j}\right|\right)^{7}<\infty .
$$

Let us assume first that $\operatorname{rank}\left(J-J_{0}\right)<\infty$. For $j \geq 1$, we define Chebyshev polynomials by recurrence relations $T_{j+1}(z)=z T_{j}(z)-T_{j-1}(z)$, and we take 
$T_{0}(z)=2, T_{1}(z)=z$. We have the so-called sum rules [1], [2], 4], 8], [9]

0) $\frac{1}{4 \pi} \int_{0}^{2 \pi} \log \frac{2 \sin \theta}{\mu^{\prime}(\theta)} d \theta=\sum_{j} \log \beta_{j}-\sum_{j} \log a_{j}$,

n) $\frac{1}{2 \pi} \int_{0}^{2 \pi} \log \frac{2 \sin \theta}{\mu^{\prime}(\theta)} \cos n \theta d \theta=\frac{1}{n} \sum_{j}\left(\beta_{j}^{n}-\beta_{j}^{-n}\right)-\frac{1}{n} \operatorname{tr}\left\{T_{n}(J)-T_{n}\left(J_{0}\right)\right\}$,

where we let $\beta_{j}=1 /\left|\zeta_{j}\right|$. Taking into account that $\sin ^{6} \theta=\left(1 / 2^{5}\right)(10-15 \cos 2 \theta+$ $6 \cos 4 \theta-\cos 6 \theta)$, we get

$$
\frac{1}{2 \pi} \int_{0}^{2 \pi} \log \frac{2 \sin \theta}{\mu^{\prime}(\theta)} \sin ^{6} \theta d \theta+\frac{1}{6} \sum_{j} F\left(\beta_{j}\right)=\frac{1}{6} \operatorname{tr}\left\{G(J)-G\left(J_{0}\right)-120 \log A\right\},
$$

where $A=\operatorname{diag}\left\{a_{k}\right\}$, and

$$
\begin{aligned}
& F(\beta)=\frac{1}{2^{5}}\left(\left(\beta^{6}-\beta^{-6}\right)-9\left(\beta^{4}-\beta^{-4}\right)+45\left(\beta^{2}-\beta^{-2}\right)-120 \log \beta\right) \\
& G(J)=\frac{1}{2^{5}}\left(T_{6}(J)-9 T_{4}(J)+45 T_{2}(J)\right)=\frac{1}{2^{5}}\left(J^{6}-15 J^{4}+90 J^{2}-110\right)
\end{aligned}
$$

Notice that $F(\beta)=C_{0}(\beta-1)^{7}+O\left((\beta-1)^{8}\right)$, where $\beta \geq 1$ and $C_{0}$ is a positive constant.

Let

$$
\Psi(a, b)=\Psi(J(a, b))=\operatorname{tr}\left\{G(J)-G\left(J_{0}\right)-120 \log A\right\} .
$$

The following lemma is a cornerstone of the proof of the theorem.

Main Lemma. Let $a=\left\{a_{k}\right\}, b=\left\{b_{k}\right\}$ and $a_{k} \neq 1, b_{k} \neq 0$, for finitely many indices only. Also let norms $\|a-1\|_{\infty},\|b\|_{\infty}$ be small enough. Then

$$
\Psi(a, b) \asymp\left(\left\|a-1||_{4}^{4}+\right\| b\left\|_{4}^{4}+\right\| \partial^{2} a\left\|_{2}^{2}+\right\| \partial^{2} b \|_{2}^{2}\right) .
$$

In particular, $\Psi(a, b) \geq 0$ for these $a$ and $b$.

The norms $\|.\|_{p}$ refer to the standard $l^{p}$-space norms. The sign " $\asymp "$ means a two-sided estimate with positive constants depending on $\|a-1\|_{\infty},\|b\|_{\infty}$. The lemma will be proved in the next section.

With the exception of this lemma, the proof of Theorem 0.1 follows a well-known scheme [4], 5]. We give only its sketch.

Proof of Theorem 0.1 . Since the absolutely continuous spectrum of $J=J(a, b)$ is the interval $[-2,2]$, we have (see [3]) that $a_{k} \rightarrow 1$ and $b_{k} \rightarrow 0$. Consequently, discarding the first $N_{0}$ rows and columns of $J$ (see [8], Section 3), we may make norms $\|a-1\|_{\infty}$ and $\|b\|_{\infty}$ small enough to apply the Main Lemma.

First, we show that condition (0.1) implies (1.1), which is equivalent to showing (0.2). Denote

$$
\Phi(J)=\Phi(\mu)=\Phi_{1}(\mu)+\Phi_{2}(\mu)=\frac{1}{2 \pi} \int_{0}^{2 \pi} \log \frac{2 \sin \theta}{\mu^{\prime}(\theta)} \sin ^{6} \theta d \theta+\frac{1}{6} \sum_{j} F\left(\frac{1}{\left|\zeta_{j}\right|}\right) .
$$

Let $a_{N}=\left\{\left(a_{N}\right)_{k}\right\}$ and $a_{N}^{\prime}=\left\{\left(a_{N}^{\prime}\right)_{k}\right\}$, where

$$
\left(a_{N}\right)_{k}=\left\{\begin{array}{ll}
a_{k}, & k \leq N, \\
1, & k>N,
\end{array} \quad\left(a_{N}^{\prime}\right)_{k}= \begin{cases}1, & k \leq N \\
a_{k}, & k>N\end{cases}\right.
$$


We also consider $b_{N}, b_{N}^{\prime}$, constructed in the same way (of course, with 1's replaced by 0's). Let $J_{N}=J\left(a_{N}, b_{N}\right)$ and $\mu_{N}$ be the corresponding measure on the unit circle. Obviously, we have that $a_{N}^{\prime}-1, b_{N}^{\prime} \rightarrow 0$ and $\partial^{2} a_{N}^{\prime}, \partial^{2} b_{N}^{\prime} \rightarrow 0$ in corresponding norms. Since

$$
\Psi(a, b)=\sum_{k} \psi\left(a_{k}, a_{k+1}, a_{k+2} ; b_{k}, b_{k+1}, b_{k+2}\right)
$$

for a function $\psi$ (see Section 22), we have that

$\left|\Psi(J)-\Psi\left(J_{N}\right)\right| \leq \Psi\left(a_{N}^{\prime}, b_{N}^{\prime}\right) \leq C_{1}\left(\left\|a_{N}^{\prime}-1\right\|\left\|_{4}^{4}+\right\| b_{N}^{\prime}\left\|_{4}^{4}+\right\| \partial^{2} a_{N}^{\prime}\left\|_{2}^{2}+\right\| \partial^{2} b_{N}^{\prime} \|_{2}^{2}\right) \rightarrow 0$, or $\Psi\left(J_{N}\right) \rightarrow \Psi(J)$. On the other hand, $\left(J_{N}-z(\zeta)\right)^{-1} \rightarrow(J-z(\zeta))^{-1}$, for $\zeta$ inside the unit disk and, consequently, $\mu_{N} \rightarrow \mu$ weakly. Using [4], Corollary 5.3 and Theorem 6.2 , we get

$$
\Phi_{1}(\mu) \leq \liminf _{N} \Phi_{1}\left(\mu_{N}\right)
$$

and

Summing up, we obtain

$$
\lim _{N \rightarrow \infty} \Phi_{2}\left(\mu_{N}\right)=\Phi_{2}(\mu)
$$

$$
\Phi(\mu) \leq \limsup _{N} \Phi\left(\mu_{N}\right)=\limsup _{N} \Psi\left(J_{N}\right)=\lim _{N \rightarrow \infty} \Psi\left(J_{N}\right)=\Psi(J) .
$$

We now show the converse, i.e., condition (1.1) yields relations (0.1). Recall (44, Proposition 8.4) that if $\mu=\mu(J)$ satisfies inequality $\mu \geq \delta m$, then

$$
\Psi(J) \leq \Phi(J),
$$

where $\delta>0$ and $m$ is the Lebesgue measure on the unit circle. We take a $J$ with the property $\Psi(J)<\infty$, and we put $\mu_{\delta}=(1-\delta) \mu+\delta m$ for $\mu=\mu(J)$ and $\delta \in(0,1)$. The measure $\mu_{\delta}$ defines a Jacobi matrix $J_{\delta}=J\left(a_{\delta}, b_{\delta}\right)$. Theorem 8.1 of [4] shows that $\Phi(J)=\lim _{\delta \rightarrow 0} \Phi\left(J_{\delta}\right)$. On the other hand (see [4], Proposition 8.4 and Theorem 8.1),

$$
\Psi(J) \leq \liminf _{\delta} \Psi\left(J_{\delta}\right)
$$

Hence,

$$
0 \leq \Psi(J) \leq \liminf _{\delta} \Psi\left(J_{\delta}\right) \leq \liminf _{\delta} \Phi\left(J_{\delta}\right)=\lim _{\delta \rightarrow 0} \Phi\left(J_{\delta}\right)=\Phi(J) .
$$

The theorem is proved.

\section{Proof of THE MAIN LEMMA}

We make a few simplifications before going into the proof. First, we drop the term $\operatorname{tr} G\left(J_{0}\right)$ in (1.3), since it is finite. Second, we discard factors $1 / 2^{5}$ (see (1.2)). Having two sequences $a=\left\{a_{k}\right\}$ and $b=\left\{b_{k}\right\}$, we denote the sequence $\left\{a_{k} b_{k}\right\}$ by $a b$. We also write $a^{ \pm}$for the sequence $\left\{a_{k \pm 1}\right\}$.

The starting point of the proof is the computation of $\operatorname{tr} J^{n}$. According to formulas from [9], Section 6.1, we have $\operatorname{tr} J^{j}=\sum_{k}\left(g_{j}\right)_{k}$, where sequences $g_{j}$ are constructed as

$$
\begin{aligned}
g_{j+1} & =h_{j}+h_{j}^{-}+b g_{j}, \\
h_{j+1} & =a^{2} \sum_{l=0}^{j} g_{j-l} g_{l}^{-}-\sum_{l=0}^{j} h_{j-l} h_{l},
\end{aligned}
$$

with $g_{0}=1, h_{0}=0$. 
We give the proof of the lemma in cases when $J=J(a, b)$ with $b=0$, and $J=J(a, b)$ with $a=1$. Since the proof in the general case follows along the same lines and is long and tedious, we omit it.

Let us consider the case when $b=0$.

Lemma 2.1. Let $a=\left\{a_{k}\right\}$ and $a_{k} \neq 1$ for finitely many indices. Then

$$
\begin{aligned}
\Psi(a)=\Psi(J(a, 0)) & =\sum_{k}\left\{-9 \lambda_{k}^{3}+3 \lambda_{k}^{2} \lambda_{k+1}+3 \lambda \lambda_{k+1}^{2}+3 \lambda_{k-1} \lambda_{k} \lambda_{k+1}\right. \\
& \left.+9 \lambda_{k}^{2}-12 \lambda \lambda_{k+1}+3 \lambda_{k-1} \lambda_{k+1}+\frac{15}{2} \lambda_{k}^{4}+O\left(\left|\lambda_{k}\right|^{5}\right)\right\},
\end{aligned}
$$

where $\lambda=a^{2}-1$.

Proof. A straightforward computation using (2.1) yields $g_{1}=g_{3}=g_{5}=0$ and

$$
\begin{aligned}
g_{2} & =a^{2}+a^{-2} \\
g_{4} & =a^{-2}\left(a^{--2}+a^{-2}+a^{2}\right)+a^{2}\left(a^{-2}+a^{2}+a^{+2}\right) \\
g_{6} & =a^{2}\left(a^{-2}\left(a^{--2}+\ldots+a^{+2}\right)+a^{2}\left(a^{-2}+\ldots+a^{+2}\right)\right. \\
& \left.+a^{+2}\left(a^{2}+\ldots+a^{++2}\right)\right) \\
& +a^{-2}\left(a ^ { - - 2 } \left(a^{---2}\right.\right. \\
& \left.\left.+\ldots+a^{2}\right)+a^{-2}\left(a^{--2}+\ldots+a^{2}\right)+a^{2}\left(a^{-2}+\ldots+a^{+2}\right)\right) .
\end{aligned}
$$

We also have $\operatorname{tr} \log A=\frac{1}{2} \sum_{k} \log \left(1+\lambda_{k}\right)$. Rewriting in terms of $\lambda=a^{2}-1$ and recalling that $\operatorname{tr} J^{j}=\sum_{k}\left(g_{j}\right)_{k}$, we get the conclusion of the lemma.

In particular, we see that

$$
\Psi(a)=\sum_{k}\left\{\psi_{1}\left(\lambda_{k}, \lambda_{k+1}, \lambda_{k+2}\right)+O\left(\left|\lambda_{k}\right|^{5}\right)\right\}
$$

where

$$
\begin{aligned}
\psi_{1}(x, y, z) & =\frac{1}{2}\left(x^{3}-20 y^{3}+z^{3}+6 x y^{2}+6 y^{2} z+6 x y z\right) \\
& +\frac{3}{2}\left(x^{2}+4 y^{2}+z^{2}-4 x y-4 y z+2 x z\right)+\frac{5}{4}\left(x^{4}+4 y^{4}+z^{4}\right) .
\end{aligned}
$$

Note also that $\lambda_{k}=2\left(a_{k}-1\right)+O\left(\left(a_{k}-1\right)^{2}\right)$.

Lemma 2.2. We have

$$
\psi_{1}(x, y, z) \asymp\left(x^{4}+y^{4}+z^{4}+(x+z-2 y)^{2}\right) .
$$

Proof. We notice that

$$
\begin{aligned}
\psi_{1}(x, y, z) & =\frac{1}{2}\left((x+z-2 y)\left(x^{2}+10 y^{2}+z^{2}+2 x y+2 y z-z x\right)\right. \\
& \left.+3(x+z-2 y)^{2}+\frac{5}{2}\left(x^{4}+4 y^{4}+z^{4}\right)\right),
\end{aligned}
$$

and the bound from above follows from the inequality $a b \leq \frac{1}{2}\left(a^{2}+b^{2}\right)$ for $a, b \geq 0$. To get the bound from below, we estimate the first term in (2.2) as

$\left|(x+z-2 y)\left(x^{2}+10 y^{2}+z^{2}+2 x y+2 y z-z x\right)\right| \leq \frac{1}{2}(x+z-2 y)^{2}+\frac{3}{2}\left(x^{4}+4 y^{4}+z^{4}\right)$,

and, consequently,

$$
\psi_{1}(x, y, z) \geq \frac{1}{2}\left(\frac{5}{2}(x+z-2 y)^{2}+\left(x^{4}+4 y^{4}+z^{4}\right)\right) .
$$

The lemma is proved. 
Now, we turn to the second case $J=J(a, b)$ with $a=1$.

Lemma 2.3. Let $b=\left\{b_{k}\right\}$ and $b_{k} \neq 0$ for finitely many indices. Then

$$
\begin{aligned}
\Psi(b)=\Psi(1, b) & =\sum_{k}\left\{b_{k}^{6}+\left(-3 b_{k}^{4}+6 b_{k} b_{k+1}^{3}+6 b_{k}^{3} b_{k+1}+6 b_{k}^{2} b_{k+1}^{2}\right)\right. \\
& \left.+\left(18 b_{k}^{2}-24 b_{k} b_{k+1}+6 b_{k-1} b_{k+1}\right)\right\} .
\end{aligned}
$$

Proof. The argument is exactly as in Lemma 2.1. We only quote expressions for $g_{5}$ and $g_{6}$ :

$$
\begin{aligned}
g_{5} & =b^{5}+\left(b^{-3}+8 b^{3}+b^{+3}+2 b^{-2} b+3 b^{-} b^{2}+3 b^{2} b^{+}+2 b b^{+2}\right) \\
& +\left(b^{--}+6 b^{-}+16 b+6 b^{+}+b^{++}\right), \\
g_{6} & =b^{6}+\left(b^{-4}+10 b^{4}+b^{+4}\right. \\
& \left.+2 b^{-3} b+3 b^{-2} b^{2}+4 b^{-} b^{3}+4 b^{3} b^{+}+3 b^{2} b^{+2}+2 b b^{+3}\right) \\
& +\left(b^{--2}+8 b^{-2}+30 b^{2}+8 b^{+2}+b^{++2}+2 b^{--} b^{-}+2 b^{--} b\right. \\
& \left.+16 b^{-} b+16 b b^{+}+2 b b^{++}+2 b^{+} b^{++}+2 b^{+} b^{-}\right)+20 .
\end{aligned}
$$

Hence, we obtain

$$
\Psi(b)=\sum_{k}\left\{\psi_{2}\left(b_{k}, b_{k+1}, b_{k+2}\right)+O\left(b_{k}^{6}\right)\right\},
$$

where

$$
\psi_{2}(x, y, z)=-3 y^{4}+\left(6 x y^{3}+6 y^{3} z+3 y^{2} x^{2}+3 y^{2} z^{2}\right)+3(x+z-2 y)^{2} .
$$

Lemma 2.4. We have

$$
\psi_{2}(x, y, z) \asymp\left(y^{4}+(x+z-2 y)^{2}\right) .
$$

Proof. One more time, the bound from above follows at once from inequality $a b \leq$ $1 /\left(p a^{p}\right)+1 /\left(q b^{q}\right)$, where $a, b \geq 0$, and $1 / p+1 / q=1$.

Furthermore, we see that

$\psi_{2}(x, y, z)=-3 y^{4}+y^{2}\left(\frac{3}{2}(x-z)^{2}+3(x+y+z)^{2}-\frac{1}{2}(x+z-2 y)^{2}\right)+3(x+z-2 y)^{2}$.

Estimating moduli of negative terms as in Lemma 2.2 and taking into account

$-3 y^{4}+3 y^{2}(x+y+z)^{2}-\frac{1}{2} y^{2}(x+z-2 y)^{2}+3(x+z-2 y)^{2} \geq \frac{1}{2}\left(y^{4}+(x+z-2 y)^{2}\right)$,

finishes the proof.

\section{REFERENCES}

[1] K. Case, Orthogonal polynomials from the viewpoint of scattering theory, J. Mathematical Phys. 15 (1974), 2166-2174. MR 50:6342

[2] K. Case, Orthogonal polynomials, II, J. Mathematical Phys. 16 (1975), 1435-1440. MR 56:2008

[3] S. Denisov, On the Nevai's conjecture and Rakhmanov's theorem for Jacobi matrices, preprint.

[4] R. Killip and B. Simon, Sum rules for Jacobi matrices and their applications to spectral theory, Annals of Math. (2) 158 (2003), 253-321.

[5] A. Laptev, S. Naboko, and O. Safronov, On new relations between spectral properties of Jacobi matrices and their coefficients, to appear.

[6] S. Molchanov, M. Novitskii, and B. Vainberg, First KdV integrals and absolutely continuous spectrum for 1-D Schrödinger operator, Comm. Math. Phys. 216 (2001), no. 1, 195-213. MR 2001k:35259 
[7] O. Safronov, The spectral measure of a Jacobi matrix in terms of the Fourier transform of the perturbation, submitted.

[8] B. Simon and A. Zlatos, Sum rules and the Szego condition for orthogonal polynomials on the real line, submitted.

[9] G. Teschl, Jacobi operators and completely integrable nonlinear lattices, Mathematical Surveys and Monographs, 72, Amer. Math. Soc., Providence, RI, 2000. MR 2001b:39019

Department of Mathematics, Box 1917, Brown University, Providence, Rhode Island 02912

E-mail address: kupin@math.brown.edu 